\title{
EFFICACY OF CERTAIN FUNGICIDES AND ANTAGONISTIC MICROORGANISMS ON MYCELIAL GROWTH OF Fusarium oxysporum ISOLATED FROM DATE PALM IN NORTH SINAI
}

\author{
AbdAllah S. Mohamed", M.Y.H. Abdalla and M.M. Sourour \\ Dept. Plant Prod., Fac. Environ. Agric. Sci., Arish Univ., Egypt
}

\begin{abstract}
In this study the efficacy of three chemical fungicides as well as five different biological control agents were tested in vitro against several isolates of Fusarium oxysporum from wilted date palm trees grown in North Sinai. The three commercial fungicides were: Tachigaren 30\% SL, Rhizolex-T 50\% WP and Dithane M-45 80\% WP. Whereas the biological control agents included three fungal species namely: Trichoderma album, T. harzianum and T. strigosum. As well as the two bacteria: Bacillus megaterium and B. subtilis. Tachigaren was the most effective fungicide in reducing the fungal growth of all tested isolates of $F$. oxysporum. Rhizolex T-50 ranked second while Dithane M-45 was the least effective fungicide in reducing the mycelial growth of the tested isolates of $F$. oxysporum in vitro. Trichoderma harzianum was the most effective biocontrol agent in inhibiting the mycelial growth of all tested F. oxysporum isolates followed by T. album and then T. strigosum. On the other hand, Bacillus megaterium and Bacillus subtilis were generally non effective in reducing mycelial growth of the tested isolates of $F$. oxysporum in vitro.
\end{abstract}

Kew word: Certain Fungicides, antagonistic microorganisms, Fusarium oxysporum isolated, date palm, North Sinai.

\section{INTRODUCTION}

The date palm (Phoenix dactylifera $\mathrm{L}$.) is a major fruit crop in most Arab countries. It has historically been connected with sustaining human life and tradition of the people in the old world as a major agricultural crop. Arab countries possess $70 \%$ of the 120 million world's date palms and are responsible for $67 \%$ of the global date production Loutfy (2010). Fusarium wilt diseases caused by Fusarium oxysporum are among the most severe plant diseases in the world. Fusarium wilts affect many plant species belonging to all the botanical families. $F$. oxysporum is one of the most common species among soil fungi in cultivated soil allover the world. It includes a large diversity of strains: saprophytic, parasitic and pathogenic.

\footnotetext{
* Corresponding author: Tel.: +201220232306

E-mail address: a.allah_saleh@aru.edu.eg
}

Fusarium oxysporum (Fo) is a ubiquitous inhabitant of soils worldwide and causes diseases such as wilt, yellows and dampingoff in different plant species Ratul and Narendra (2010). The fungus can invade plant's roots with its conidial germ tube or mycelium. The roots can be infected directly through the root tips, wounds in the roots or at the formation point of lateral roots. Mycelium enters the xylem vessels branches and produces micro-conidia which are carried upward in the sap stream. Due to the growth of the fungus within the plant's vascular tissue, the plant's water supply is greatly affected. This lack of water induces the leaves stomata to close, the leaves wilt and the plant eventually dies. Infection usually leads to chlorosis, leaf wilting and browning of the vascular system. Several procedures have been attempted for managing 
the fusarium wilt disease in the greenhouse and in field, Ioannou (1999). Bayoud of date palm caused by the fungus Fusarium oxysporum f.sp. albedinis (Foa) is the most important disease of this crop. It is currently confined to Morocco and the western and central regions of Algeria, having destroyed approximately 15 million trees since it was first discovered, sometime before 1870, Djerbi (1983).

In this research we studied the effects of some chemical fungicides as well as biofungicides, on the growth of several isolates of Fusarium oxysporum isolated from date palm grown in North Sinai.

\section{MATERIALS AND METHODS}

\section{Isolation of Fungal Pathogens}

Fusarium oxysporum cultures were isolated from infected roots of date palm trees showing wilt, drying, yellow leaves and progressive die back from different districts located in four different geographic regions in North Sinai. Root samples were washed carefully with tap water to remove the adhering soil. Diseased root samples were cut into pieces ( $1 \mathrm{~cm}$ long). Surface sterilized with dipping in $0.5 \%$ sodium hypochlorite solution for 2 minutes, then washed thoroughly with several changes of sterile distilled water. The cuted pieces were dried between sterile filter papers. The surface sterilized pieces were transferred to Petri dished containing acidified potato dextrose agar medium (PDA) then incubated at $25 \pm 2{ }^{\circ} \mathrm{C}$ for two weeks then inspected for fungal growth, Rashed (1991), El-Morsi (1999), Abdalla et al. (2001), El-Morsi (2004) and Baraka et al. (2011).

\section{Fusarium oxysporum Control In vitro}

Effect of chemical fungicides on the linear growth of $F$. oxysporum

Three fungicides: Hymexazol (Tachigaren $30 \%$ SL), Tolclofos- methyl (Rhizolex-T
$50 \%$ WP) and Mancozeb (Dithane M-45 $80 \%$ WP) were tested individually at different concentration $(10,50,100,500$ and $1000 \mathrm{ppm})$. The effects of the fungicides were assayed by using the poisoned food technique according to the method described by Abdalla et al. (1992). Fungicides were added to autoclaved PDA medium before dispensing medium in Petri plates, when temperature of the medium was about $50^{\circ} \mathrm{C}$. Control treatment was PDA medium without fungicides additions.

Disks $(5 \mathrm{~mm})$ in diameter from 7 days old cultures of the tested fungus were transferred to the center of Petri plates then of the plates were incubated at $27 \pm 2{ }^{\circ} \mathrm{C}$ for 16 days. After 4, 8, 12 and 16 days of incubation the linear growth of the tested was assessed by measuring two perpendicular diameters in $\mathrm{cm}$ and the average was recorded.

\section{Biological control}

The Trichoderma album isolate was isolated from the commercial biological control product called "Bio zeid", Trichoderma strigosum and Trichoderma harzianum were isolated by one gram of dry soil from each sample was added to test tubes containing $9 \mathrm{ml}$ sterile water and each test tube was shaken periodically for approximately 15 minutes. After shaking one $\mathrm{ml}$ of suspension was added to test tube containing $9 \mathrm{ml}$ sterile water, and the process was repeated to make serial dilutions up to 1:10000 for each fungus isolation. Aliquots $1 \mathrm{ml}$ of serial dilutions was spread on APDA plates. All plates were incubated at $25^{\circ} \mathrm{C}$. After 2-7 days the isolated microorganisms, were identified according to their morphological characters, Samuels and Hebbar (2015). Selected microorganisms were purified and transferred on to APDA slants; pure cultures were stored at $4^{\circ} \mathrm{C}$ for further studies. The antagonistic fungi were identified at Unit of Identification of Microorganisms, Plant Pathology Research Institute, Agricultural Research Center, using BIOLOG ${ }^{\mathrm{TM}}$ system. 
The Bacillus megaterium culture was isolated from the commercial biological control product called "Bio Arc" and the Bacillus subtilis culture was isolated from the commercial biological control product called "Clean Root".

The biological control agents were tested against $F$. oxysporum isolates in vitro using acidified potato dextrose agar (APDA) medium in $9 \mathrm{~cm}$ diameter Petri plates. Five $\mathrm{mm}$ diameter disc of a four days old fungal growth of the tested $F$. oxysporum isolates were placed at about two $\mathrm{cm}$ from the edge of the plates.

On the opposite side of the Petri plates a five $\mathrm{mm}$ disc of the tested antagonistic fungi was placed. Plates were incubated at $25^{\circ} \mathrm{C}$ and kept under daily observation and data were recorded one week later as linear growth of the $F$. oxysporum cultures as described by Abd El-Moity et al. (1993) and Perveen and Bokhari (2012). The bacterium was maintained on PDA medium at $33 \pm 2^{\circ} \mathrm{C}$. The Bacillus sp. isolate was tested against $F$. oxysporum using PDA medium in $9 \mathrm{~cm}$ diameter Petri plates. 5 mm diameter discs of the tested fungus was placed two $\mathrm{cm}$ from the edge of the plate. On the opposite side of the Petri plates at about $2 \mathrm{~cm}$ from the edge a streak of the tested antagonistic bacterium was placed as suggested by Agarwal et al. (2011). These experiments were repeated twice and data were recorded.

\section{RESULTS AND DISCUSION}

\section{Fusarium oxysporum Control In vitro}

\section{Effect of chemical fungicides on linear growth of $F$. oxysporum}

In vitro studies Table 1 indicate that Tachigaren $30 \%$ SL was very effective in controlling radial growth of $F$. oxysporum at $1000 \mathrm{ppm}$ on tested isolates $\left(\mathrm{D}_{2}, \mathrm{E}_{1}\right.$ and $\mathrm{F}_{7}$ ) 16 days after incubation, but it showed little effect on isolate $B_{2} 16$ days after incubation. Rhizolex-T 50\% WP ranked second in controlling radial growth of $F$. oxysporum isolates $\left(\mathrm{E}_{1}\right.$ and $\left.\mathrm{F}_{7}\right)$. It was less effective on linear growth of $F$. oxysporum isolate $\mathrm{D}_{2}$, and showed no significant effect on isolate $\mathrm{B}_{2}$ after 16 days of incubation. The fungicide Dithane M-45 $80 \%$ WP was the least effective fungicide in controlling radial growth of Fusarium oxysporum isolates $\left(\mathrm{B}_{2}, \mathrm{D}_{2}, \mathrm{E}_{1}\right.$ and $\left.\mathrm{F}_{7}\right)$ at all tested concentrations 16 days after incubation on APDA medium.

These results are in agreement with those obtained by Haggag and El-Gamal (2012) who found that Tachigaren was the most effective fungicide in reducing the mycelial growth of Fusarium solani. Similarly, Song et al. (2004) demonstrated that Prochloraz and Carbendzim were more efficient in reducing mycelial growth of $F$. oxysporum f.sp. lycopersici compared with Tachigaren and Dithane M-45.

In vitro antagonism of microorganisms on the pathogen Fusarium oxysporum

All tested isolates of Trichoderma strigosum, T. harzianum and T. album suppressed the growth of $F$. oxysporum isolates $\left(B_{2}, D_{2}, E_{1}\right.$ and $\left.F_{7}\right)$, this inhibition occurred before any direct mycelial interaction took place (Table 2 and Plate 1).

Trichoderma strigosum isolate significantly suppressed the growth of $F$. oxysporum isolates and it subsequently mycoparasitised the pathogen expect in case of isolate $B_{2}$, T. strigosum did not overgrow it.

Trichoderma harzianum isolate also significant suppressed the growth of $F$. oxysporum isolates and it subsequently mycoparasitised the pathogen expect in case of isolate $\mathrm{B}_{2}, T$. harzianum did not overgrow it.

Trichoderma album isolates was significantly inhibiting the growth of the pathogen $F$. oxysporum isolates and it subsequently mycoparasitised the pathogen, expect isolate $B_{2}$. 
Table (1): Effect of five concentrations of three fungicides "Tachigaren, Rhizolex and Dithane M-45" on the linear growth $(\mathrm{mm})$ of $F$. oxysporum in vitro isolate $\left(\mathrm{B}_{2}\right.$, $D_{2}, E_{1}$ and $\left.F_{7}\right) 4,8,12$ and 16 days after incubation.

\begin{tabular}{|c|c|c|c|c|c|c|c|}
\hline \multirow{2}{*}{\multicolumn{4}{|c|}{ Treatments }} & \multicolumn{4}{|c|}{$\begin{array}{c}\text { Rate growth rate of Fusarium oxysporum isolates } \\
\text { after incubation (day) }\end{array}$} \\
\hline & & & & 4 days & 8 days & 12 days & 16 days \\
\hline \multirow{16}{*}{ 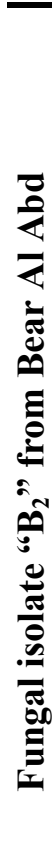 } & \multirow{7}{*}{ Tachigaren 30\% SL } & \multicolumn{2}{|c|}{ Control } & $30.00 \mathrm{a}$ & $50.00 \mathrm{fgh}$ & $90.00 \mathrm{a}$ & $90.00 \mathrm{a}$ \\
\hline & & 10 & ppm & $21.50 \mathrm{ghi}$ & $44.00 \mathrm{jklm}$ & $60.50 \mathrm{jkl}$ & $76.15 \mathrm{ij}$ \\
\hline & & & ppm & $19.75 \mathrm{ijkl}$ & $42.87 \mathrm{lmn}$ & $56.50 \mathrm{mn}$ & $78.75 \mathrm{hi}$ \\
\hline & & 100 & ppm & $16.62 n$ & $37.25 \mathrm{pq}$ & 50.25 op & $82.00 \mathrm{fgh}$ \\
\hline & & 500 & ppm & $4.50 \quad \mathrm{vw}$ & $13.50 \mathrm{DE}$ & $27.25 \mathrm{z}$ & $22.60 \mathrm{yz}$ \\
\hline & & 1000 & ppm & $3.62 \mathrm{~W}$ & $11.75 \mathrm{E}$ & $16.75 \mathrm{~B}$ & $18.87 \mathrm{~A}$ \\
\hline & & Cont & & $30.00 \mathrm{a}$ & $50.00 \mathrm{fgh}$ & $90.00 \mathrm{a}$ & $90.00 \mathrm{a}$ \\
\hline & \multirow{6}{*}{ Rhizolex-T 50 \% WP } & 10 & ppm & $23.00 \mathrm{defg}$ & $41.00 \mathrm{mno}$ & $71.00 \mathrm{hi}$ & $90.00 \mathrm{a}$ \\
\hline & & & ppm & $17.00 \mathrm{mn}$ & $34.00 \mathrm{rs}$ & $61.50 \mathrm{jk}$ & $90.00 \mathrm{a}$ \\
\hline & & 100 & ppm & $12.25 \mathrm{q}$ & $25.50 \mathrm{wx}$ & $46.00 \mathrm{qrs}$ & $72.82 \mathrm{kl}$ \\
\hline & & 500 & ppm & $9.00 \mathrm{tu}$ & $20.50 \mathrm{zA}$ & $41.50 \mathrm{tu}$ & $62.75 \mathrm{p}$ \\
\hline & & 1000 & ppm & $5.75 \mathrm{v}$ & $14.50 \mathrm{CDE}$ & 32.75 xy & $55.62 \mathrm{qr}$ \\
\hline & & Cont & & $30.00 \mathrm{a}$ & $50.00 \mathrm{fgh}$ & $90.00 \mathrm{a}$ & $90.00 \mathrm{a}$ \\
\hline & \multirow{7}{*}{$\begin{array}{l}\text { Dithane M-45 } \\
80 \% \text { WP }\end{array}$} & 10 & ppm & $22.25 \mathrm{efgh}$ & $46.28 \mathrm{ij}$ & $69.25 \mathrm{hi}$ & $88.75 \mathrm{abc}$ \\
\hline & & 50 & ppm & $19.25 \mathrm{kl}$ & 40.56 nno & $59.75 \mathrm{jklm}$ & $80.50 \mathrm{gh}$ \\
\hline & & 100 & ppm & 16.18 no & $36.81 \mathrm{pqr}$ & 53.43 no & $86.50 \mathrm{bcd}$ \\
\hline \multirow{20}{*}{ 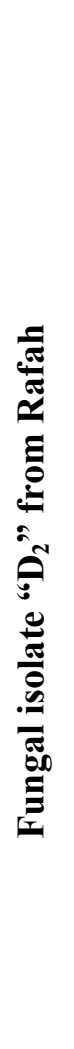 } & & 500 & ppm & $14.31 \mathrm{p}$ & $31.93 \mathrm{stu}$ & 50.43 op & $84.25 \mathrm{def}$ \\
\hline & & 1000 & ppm & 11.96 qrs & $23.93 \mathrm{xy}$ & $46.50 \mathrm{qr}$ & $89.17 \mathrm{ab}$ \\
\hline & & Cont & & $30.00 \mathrm{a}$ & $50.00 \mathrm{fgh}$ & $90.00 \mathrm{a}$ & $90.00 \mathrm{a}$ \\
\hline & & 10 & ppm & 14.50 op & 30.50 tu & $37.25 \mathrm{vw}$ & $53.10 \mathrm{rs}$ \\
\hline & \multirow[t]{6}{*}{ Tachigaren 30\% SL } & 50 & ppm & $12.06 \mathrm{qr}$ & $26.25 \mathrm{wx}$ & $38.25 \mathrm{uv}$ & $49.62 \mathrm{t}$ \\
\hline & & 100 & ppm & $10.21 \mathrm{st}$ & 24.50 wxy & 34.25 wxy & $47.82 \mathrm{tu}$ \\
\hline & & 500 & ppm & $0.00 \mathrm{x}$ & $0.00 \quad \mathrm{~F}$ & $0.00 \quad \mathrm{D}$ & $2.50 \quad \mathrm{~B}$ \\
\hline & & 1000 & ppm & $0.00 \mathrm{x}$ & $0.00 \quad \mathrm{~F}$ & $0.00 \quad \mathrm{D}$ & $0.00 \quad \mathrm{~B}$ \\
\hline & & Cont & & $30.00 \mathrm{a}$ & $55.00 \mathrm{~cd}$ & $82.00 \mathrm{c}$ & $90.00 \mathrm{a}$ \\
\hline & & 10 & ppm & $24.55 \mathrm{~cd}$ & $54.25 \mathrm{de}$ & $80.25 \mathrm{~cd}$ & $90.00 \mathrm{a}$ \\
\hline & \multirow{5}{*}{ Rhizolex-T 50 \% WP } & 50 & ppm & $10.28 \mathrm{rst}$ & $31.25 \mathrm{stu}$ & $49.00 \mathrm{pq}$ & $75.45 \mathrm{jk}$ \\
\hline & & 100 & ppm & $9.08 \mathrm{tu}$ & $25.57 \mathrm{wx}$ & $38.00 \mathrm{v}$ & $56.45 \mathrm{q}$ \\
\hline & & 500 & ppm & $0.00 \mathrm{x}$ & 16.00 BCD & $18.75 \mathrm{~B}$ & $42.82 \mathrm{~V}$ \\
\hline & & 1000 & ppm & $0.00 \quad x$ & $0.00 \quad F$ & $13.00 \mathrm{C}$ & $21.77 \mathrm{zA}$ \\
\hline & & Cont & & $30.00 \mathrm{a}$ & $55.00 \mathrm{~cd}$ & $82.00 \mathrm{c}$ & $90.00 \mathrm{a}$ \\
\hline & \multirow{5}{*}{$\begin{array}{l}\text { Dithane M-45 } \\
80 \% \text { WP }\end{array}$} & 10 & ppm & $25.01 \mathrm{c}$ & $50.00 \mathrm{fgh}$ & $77.00 \mathrm{de}$ & $89.50 \mathrm{ab}$ \\
\hline & & 50 & ppm & $21.20 \mathrm{hij}$ & $42.50 \mathrm{lmn}$ & $69.50 \mathrm{hi}$ & $89.75 \mathrm{ab}$ \\
\hline & & 100 & ppm & $19.77 \mathrm{ijkl}$ & 38.25 op & $59.50 \mathrm{jklm}$ & $85.25 \mathrm{def}$ \\
\hline & & 500 & ppm & $19.55 \mathrm{jkl}$ & $42.00 \mathrm{lmn}$ & 53.25 no & $71.37 \mathrm{~lm}$ \\
\hline & & 1000 & ppm & 14.65 op & $32.75 \mathrm{st}$ & $44.50 \mathrm{rst}$ & 65.00 ор \\
\hline
\end{tabular}


Table (1): Con. Effect of five concentrations of three fungicides "Tachigaren, Rhizolex and Dithane M-45" on the linear growth $(\mathrm{mm})$ of $F$. oxysporum in vitro isolate $\left(B_{2}, D_{2}, E_{1}\right.$ and $\left.F_{7}\right) 4,8,12$ and 16 days after incubation.

\begin{tabular}{|c|c|c|c|c|c|c|c|}
\hline \multirow{2}{*}{\multicolumn{4}{|c|}{ Treatments }} & \multicolumn{4}{|c|}{$\begin{array}{c}\text { Growth rate of Fusarium oxysporum isolates after } \\
\text { incubation (day) }\end{array}$} \\
\hline & & & & 4 days & \multirow{2}{*}{$\begin{array}{l}\text { 8 days } \\
65.00 \mathrm{a}\end{array}$} & \multirow{2}{*}{$\frac{12 \text { days }}{90.00 \mathrm{a}}$} & 16 days \\
\hline \multirow{12}{*}{ 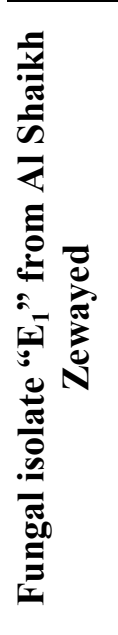 } & \multirow{5}{*}{$\begin{array}{l}\text { Tachigaren } \\
\text { 30\% SL }\end{array}$} & \multicolumn{2}{|c|}{ Control } & $30.00 \mathrm{a}$ & & & $90.00 \mathrm{a}$ \\
\hline & & & ppm & $22.50 \mathrm{efgh}$ & 48.50 ghi & $68.00 \mathrm{i}$ & 85.75 cde \\
\hline & & & ppm & $18.50 \mathrm{~lm}$ & 41.25 lmno & $58.50 \mathrm{klm}$ & $82.95 \mathrm{efg}$ \\
\hline & & 100 & ppm & 19.001 & $34.25 \mathrm{qrs}$ & $53.00 \mathrm{o}$ & $75.45 \mathrm{jk}$ \\
\hline & & 500 & ppm & $3.42 \mathrm{w}$ & 14.25 DE & $18.75 \mathrm{~B}$ & $25.50 \mathrm{y}$ \\
\hline & \multirow{12}{*}{$\begin{array}{l}\text { Dithane M-45 } \\
80 \% \text { WP }\end{array}$} & 1000 & ppm & $0.00 \mathrm{x}$ & $0.00 \quad F$ & $0.00 \quad \mathrm{D}$ & $0.00 \quad \mathrm{~B}$ \\
\hline & & Contr & & $30.00 \mathrm{a}$ & $65.00 \mathrm{a}$ & $90.00 \mathrm{a}$ & $90.00 \mathrm{a}$ \\
\hline & & & ppm & 20.90 hijk & $47.50 \mathrm{hi}$ & $57.75 \mathrm{~lm}$ & $72.50 \mathrm{kl}$ \\
\hline & & & ppm & $11.42 \mathrm{qrs}$ & $29.50 \mathrm{uv}$ & $43.25 \mathrm{rst}$ & $69.00 \mathrm{mn}$ \\
\hline & & 100 & ppm & $8.87 \mathrm{tu}$ & $19.00 \mathrm{zAB}$ & 33.00 xy & $44.67 \mathrm{uv}$ \\
\hline & & 500 & ppm & $0.00 \mathrm{x}$ & $14.50 \mathrm{CDE}$ & $22.37 \mathrm{~A}$ & $35.40 \mathrm{w}$ \\
\hline & & 1000 & ppm & $0.00 \mathrm{x}$ & $0.00 \quad F$ & $0.00 \quad \mathrm{D}$ & $20.90 \mathrm{zA}$ \\
\hline \multirow{11}{*}{ 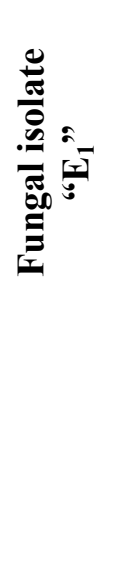 } & & Col & ntrol & $30.00 \mathrm{a}$ & $65.00 \mathrm{a}$ & $90.00 \mathrm{a}$ & $90.00 \mathrm{a}$ \\
\hline & & & ppm & $24.87 \mathrm{c}$ & $52.05 \mathrm{def}$ & 75.50 ef & $90.00 \mathrm{a}$ \\
\hline & & & ppm & 19.071 & $44.12 \mathrm{jkl}$ & $62.75 \mathrm{j}$ & $90.00 \mathrm{a}$ \\
\hline & & & ppm & 21.42 ghi & $43.00 \mathrm{klmn}$ & $61.50 \mathrm{jk}$ & $90.00 \mathrm{a}$ \\
\hline & & & ppm & $22.20 \mathrm{fgh}$ & $47.25 \mathrm{hi}$ & 75.75 ef & $90.00 \mathrm{a}$ \\
\hline & & 1000 & ppm & 23.05 defg & 48.25 ghi & $77.50 \mathrm{de}$ & $90.00 \mathrm{a}$ \\
\hline & & Col & ntrol & $30.00 \mathrm{a}$ & $63.00 \mathrm{a}$ & $90.00 \mathrm{a}$ & $90.00 \mathrm{a}$ \\
\hline & & & ppm & $10.50 \mathrm{qrst}$ & $27.02 \mathrm{vw}$ & $43.00 \mathrm{st}$ & $71.00 \mathrm{~lm}$ \\
\hline & Tachigaren & & ppm & $9.57 \mathrm{tu}$ & $21.50 \mathrm{yz}$ & $35.50 \mathrm{vwx}$ & $56.25 \mathrm{qr}$ \\
\hline & & 100 & ppm & 7.85 & $17.50 \mathrm{ABC}$ & $31.25 \mathrm{y}$ & $31.00 \mathrm{x}$ \\
\hline & & 500 & ppm & 0.00 & $0.00 \quad F$ & $0.00 \quad \mathrm{D}$ & $0.00 \quad \mathrm{~B}$ \\
\hline \multirow{14}{*}{ 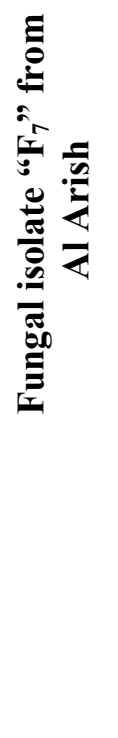 } & & 1000 & ppm & $0.00 \mathrm{x}$ & $0.00 \quad F$ & $0.00 \quad \mathrm{D}$ & $0.00 \quad \mathrm{~B}$ \\
\hline & & Co & ntrol & $29.00 \mathrm{a}$ & $63.00 \mathrm{a}$ & $90.00 \mathrm{a}$ & $90.00 \mathrm{a}$ \\
\hline & & & ppm & $27.02 \mathrm{~b}$ & $59.00 \mathrm{~b}$ & $77.25 \mathrm{de}$ & $90.00 \mathrm{a}$ \\
\hline & $\begin{array}{l}\text { Rhizolex-T } \\
50 \text { WP }\end{array}$ & 50 & ppm & 15.22 nop & $42.50 \mathrm{lmn}$ & $71.75 \mathrm{gh}$ & $90.00 \mathrm{a}$ \\
\hline & & 100 & ppm & 11.45 qrs & $26.00 \mathrm{wx}$ & $44.00 \mathrm{rst}$ & 66.62 no \\
\hline & & & ppm & $0.00 \quad x$ & $12.85 \mathrm{E}$ & $27.50 \mathrm{z}$ & $50.00 \mathrm{st}$ \\
\hline & & 1000 & ppm & 0.00 & 0.00 & $0.00 \quad \mathrm{D}$ & $21.10 \mathrm{zA}$ \\
\hline & & Col & ntrol & $29.00 \mathrm{a}$ & $63.00 \mathrm{a}$ & $90.00 \mathrm{a}$ & $90.00 \mathrm{a}$ \\
\hline & & 10 & ppm & $27.07 \mathrm{~b}$ & 51.25 efg & $88.50 \mathrm{ab}$ & $90.00 \mathrm{a}$ \\
\hline & Dithane M-45 & 50 & ppm & $23.00 \mathrm{defg}$ & $50.25 \mathrm{fgh}$ & $72.50 \mathrm{fgh}$ & $90.00 \mathrm{a}$ \\
\hline & & 100 & ppm & 23.50 cdef & $46.00 \mathrm{ijk}$ & 74.75 efg & $90.00 \mathrm{a}$ \\
\hline & & 500 & ppm & 24.00 cde & $58.00 \mathrm{bc}$ & $86.25 \mathrm{~b}$ & $90.00 \mathrm{a}$ \\
\hline & & 1000 & ppm & $27.00 \mathrm{~b}$ & $65.25 \mathrm{a}$ & $90.00 \mathrm{a}$ & $90.00 \mathrm{a}$ \\
\hline & LSD 0.05 & & & 1.777 & 3.014 & 3.342 & 3.257 \\
\hline
\end{tabular}


Table (2): The antagonistic effect of the bio-control agent: $T$. strigosum, T. harzianum, $T$. album, Bacillus megaterium and $B$. subtilis against $F$. oxysporum isolates growth $(\mathrm{mm})$ in vitro 8 days after incubation.

\begin{tabular}{|c|c|c|c|c|}
\hline \multirow{2}{*}{$\begin{array}{c}\text { Origin of } \\
\text { fungal isolates }\end{array}$} & \multirow[t]{2}{*}{ Treatment } & \multicolumn{3}{|c|}{ Main linear growth of $F$. oxysporum (mm) } \\
\hline & & $\begin{array}{c}\text { Microorganism against } \\
\text { F. oxysporum }\end{array}$ & Control & $\begin{array}{c}* \text { PGI } \\
(\%) \\
\end{array}$ \\
\hline \multirow{5}{*}{ 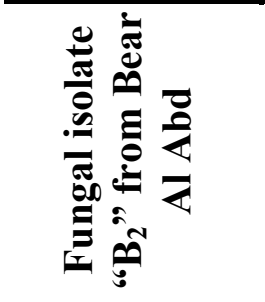 } & T. strigosum & $25.50 \mathrm{e}$ & \multirow{5}{*}{90} & 71.66 \\
\hline & T. harzianum & $26.75 \mathrm{e}$ & & 70.27 \\
\hline & T. album & $19.72 \mathrm{fgh}$ & & 78.17 \\
\hline & B. megaterium & $50.00 \mathrm{a}$ & & 44.44 \\
\hline & B. subtilis & $45.00 \mathrm{~b}$ & & 50 \\
\hline \multirow{5}{*}{ 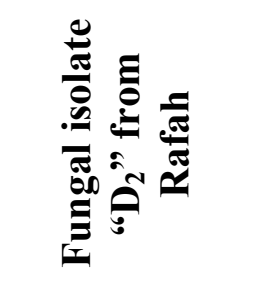 } & T. strigosum & $25.05 \mathrm{e}$ & \multirow{5}{*}{90} & 72.16 \\
\hline & T. harzianum & $18.35 \mathrm{gh}$ & & 79.61 \\
\hline & T. album & $24.82 \mathrm{e}$ & & 72.42 \\
\hline & B. megaterium & $45.00 \mathrm{~b}$ & & 50 \\
\hline & B. subtilis & $37.00 \mathrm{~d}$ & & 5.88 \\
\hline \multirow{5}{*}{ 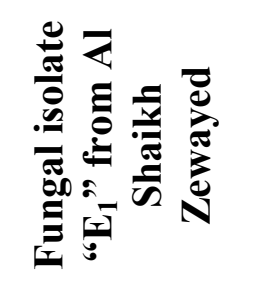 } & T. strigosum & $26.00 \mathrm{e}$ & \multirow{5}{*}{90} & 71.11 \\
\hline & T. harzianum & $18.72 \mathrm{gh}$ & & 79.20 \\
\hline & T. album & $20.87 \mathrm{fg}$ & & 76.81 \\
\hline & B. megaterium & $48.00 \mathrm{a}$ & & 46.66 \\
\hline & B. subtilis & $42.00 \mathrm{c}$ & & 53.33 \\
\hline \multirow{6}{*}{ 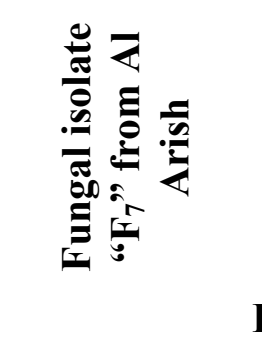 } & T. strigosum & $22.07 \mathrm{f}$ & \multirow{6}{*}{90} & 75.47 \\
\hline & T. harzianum & $16.91 \mathrm{~h}$ & & 81.21 \\
\hline & T. album & $17.97 \mathrm{gh}$ & & 80.03 \\
\hline & B. megaterium & $48.00 \mathrm{a}$ & & 46.66 \\
\hline & B. subtilis & $45.00 \mathrm{~b}$ & & 50 \\
\hline & 0.05 & & & \\
\hline
\end{tabular}

* PGI (Percent Growth Inhibition) $=$ Colony growth in control plate - Colony growth in intersecting plate Colony growth in control plate $\times 100$

- Each treatment represented the mean of six replicates for two experiment.

Similar results were obtained by Perveen and Bokhari (2012). They studied the antagonistic potential of $T$. harzianum and $T$. viride against $F$. oxysporum isolated from date palm. They concluded that all tested isolates of Trichoderma showed appreciable inhibition for the mycelial growth of $F$. oxysporum.

Bacillus megaterium was non-significant in controlling radial growth of all tested Fusarium oxysporum isolates 8 days after incubation, no inhibition zones were observed with any of these isolates after incubation on PDA medium.

Bacillus subtilis was very effective in controlling radial growth of $F$. oxysporum isolates $\left(B_{2}, D_{2}\right.$ and $\left.E_{1}\right)$ except isolate $F_{7}$ it was non-significant in controlling radial growth of the pathogen after incubation on PDA medium.

Several researchers have investigated bio-control strategies using various bacterial species to control $F$. oxysporum f.sp. albedinis 


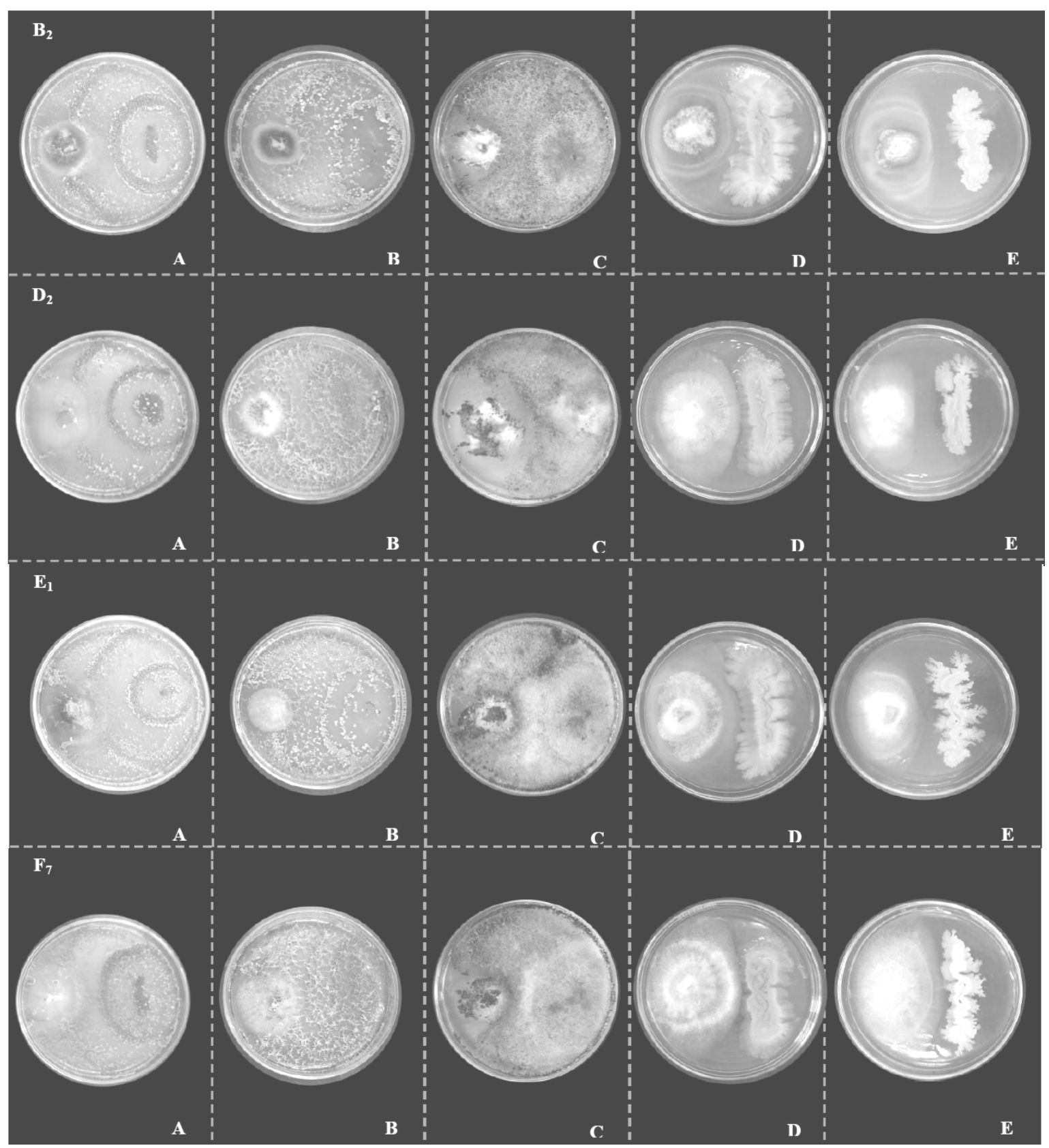

Plate (1): The antagonistic effect of T. strigosum (A), T. harzianum (B), T. album (C), B.megaterium (D) and B. subtilis (E) against $F$. oxysporum isolates growth in vitro after 8 days incubation

which cause the most destructive disease of date palm, called Bayoud disease. For instance, Dihazi et al., (2012), found that Bacillus amyloliquefaciens and Burkholderia cepacia significantly inhibited growth and sporulation of $F$. oxysporum f.sp. albedinis. Also, selected three bacterial species namely: B. pumilus, Rahnella aquatilis,
Bacillus oereus for their high inhibition toward mycelial growth of $F$. oxysporum f.sp. albedinis.

These success in finding biological control agents against $F$. oxysporum are providing hope for establishing biocontrol strategy against Fusarium wilt of date palm. 


\section{REFERENCES}

Abd El-Moity, S.M.H.; Abdalla, M.Y. and Shehata, M.R.A. (1993). Evaluation of certain microorganisms for the biological control of chocolate spot disease on faba bean. Bull. Suez Canal Univ. Appl. Sci., 2: 17-32.

Abdalla, M.Y.; Ibrahim, G.H.; AlMihanna, A.A. and Al-Rokaibah, A.A. (2001). Brown leaf spot of date palm in Saudi Arabia and the Influence of leaf epicuticular wax on disease severity. J. Adv. Agric. Res., 6 (3): 585-594.

Abdalla, M.Y.; Kassem, F.A.; Shehata, M.R.A. and Ahmed, A.H. (1992). Etiology and control of offshoot heart rot of date palm in Al-Arish. Bull, of Suez Canal Univ. Appl. Sci., 1: 246-259.

Agarwal, T.; Abhiniti, M.; Manish, B. and Trivedi, P.C. (2011). In vitro interaction of Trichoderma isolates against Aspergillus niger, Chaetomium sp. and Penicilium sp. Indian J. Fundamental and Appl. Life Sci., 1 (3): 125-128.

Baraka, M.A.; Radwan, F.M. and Arafat, K.H. (2011). Studies on fungal root diseases of date palm and its control. J. Biol. Chem. Environ. Sci., 6 (2): 319-337.

Dihazi, A.; Jaiti, F.; Wafataktak.; Kilani, F.O.; Jaoua, S.; Driouich, A.; Baaziz, M.; Daayf, F. and Serghini, M.A. (2012). Use of two bacteria for biological control of bayoud disease caused by Fusarium oxysporum in date palm (Phoenix dactylifera L.) seedlings. Plant Physiol Biochem. Doi:10.1016/j. plaphy. 2012.03.003.

Djerbi, M. (1983). Diseases of the date palm (Phoenix dactylifera). Regional project for palm and dates Research center in Near East and North Africa. Baghdad, Iraq, FAO, 106.

El-Morsi, M.A.E. (1999). Studies on certain fungal diseases of date palm in
New Valley. M.Sc. Thesis, Fac. Agric., Assiut Uni., 110.

El-Morsi, M.A.E. (2004). Studies on certain fungal diseases of date palm offshots in New Valley. Fac. Agric., Assiut Uni., 120.

Haggag, Karima H.E. and El-Gamal Nadia G. (2012). In vitro study on Fusarium solani and Rhizoctonai solani isolates causing the damping off and root rot diseases in tomato. Cent. Agric. Pest. Lib., Agric. Res. Cent., Dokki, Giza, Egypt. Nat. and Sci., 10 (11): 16-25.

Ioannou, N. (1999). Management of soil borne pathogens of tomato with soil solarization. Technical Bulletin., Agric. Res. Inst., Nicosia, Cyprus (20): 5-9.

Loutfy, I.E. (2010) Degradation of date palm trees and date production in arab countries: causes and potential rehabilitation. Aust. J. Basic and Appl. Sci., 4 (8): 3998-4010.

Perveen, K. and Bokhari, N.A. (2012). Antagonistic activity of Trichoderma harzianum and Trichoderma viride isolated from soil of date palm field against Fusarium oxysporum. African J. Micro. Res., 6 (13): 3348-3353.

Rashed, M.F. (1991). Pathological studies on fungi causing deterioration of date palm off-shoots in Egypt M.Sc. Thesis, Fac., Agric. Cairo Uni., 90.

Ratul, S. and Narendra, K. (2010). Molecular detection and identification of Fusarium oxysporum. DOI 10.1007/9783-642-05042-8_7,Springer-Verlag Berlin Heidelberg, 1-28.

Samuels, G.J. and Hebbar, P.K. (2015). Trichoderma: Identification and agricultural applications. APS Press, st. Panl. MN., 204.

Song, W.; Zhou, L.; Yang, C.; Cao, X.; Zhang, L. and Liu, X. (2004). Tomato Fusarium wilt and its chemical control strategies in a hydroponic system. Crop Port., 23: 243-247. 


$$
\text { الملخص العربى }
$$

تأثير بعض المسببات الفطرية والكائنات البيولوجية المضادة علي نمو فطر الفيوزاريوم أوكسيسبورم

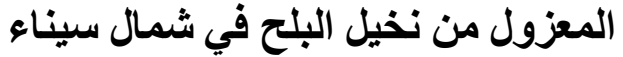

\section{عبدالله صالح محمد، محمد ياسر حسن عبدالله، محمد محمود سرور}

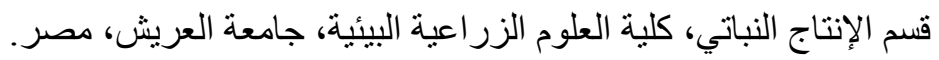

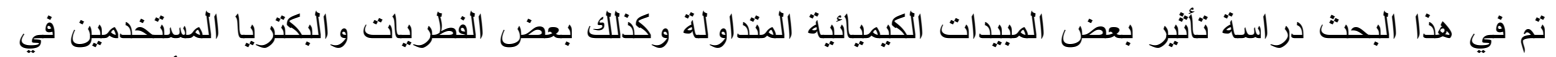

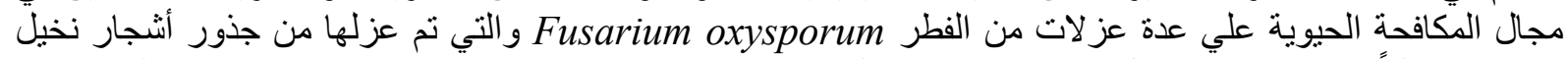

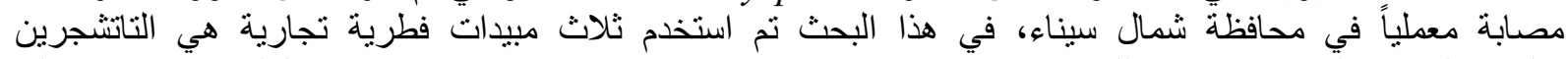

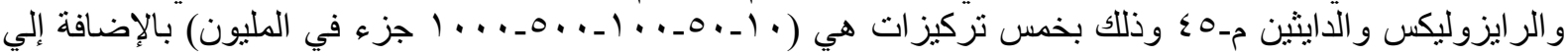

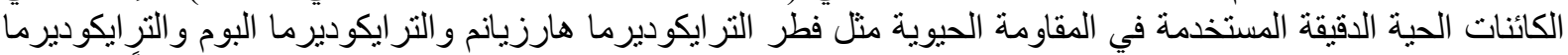

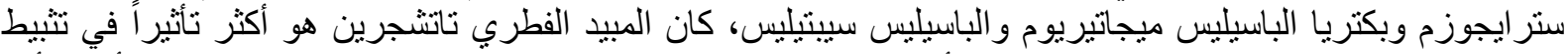

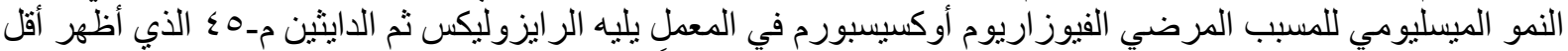

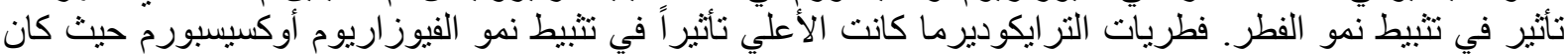

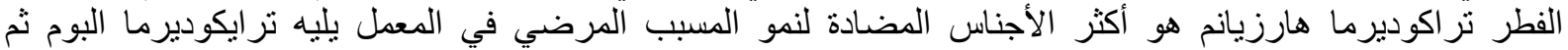

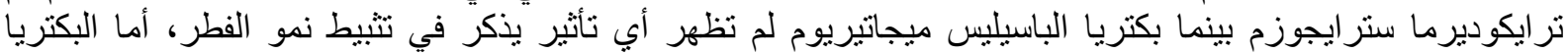

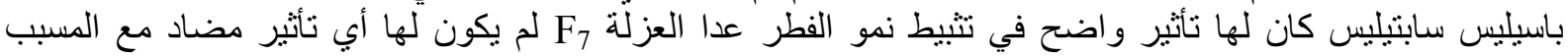
المرضي.

الكلمات الاسترشادية: المسببات الفطرية، الكائنات البيولوجية المضادة، فطر الفيوزاريوم أوكسيسبورم، نخيل البلح، شمال 
Mohamed, et al. 